\title{
CAD Fractal Art Pattern Design and Evaluation Based on RBF Network Algorithm
}

\author{
Lina Liu and Danjun Zhu \\ School of Art \& Design, Henan University of Science and Technology, Luoyang, 471000, China \\ Correspondence should be addressed to Danjun Zhu; 9903680@haust.edu.cn
}

Received 16 November 2021; Revised 17 December 2021; Accepted 24 December 2021; Published 22 January 2022

Academic Editor: Qiangyi Li

Copyright (c) 2022 Lina Liu and Danjun Zhu. This is an open access article distributed under the Creative Commons Attribution License, which permits unrestricted use, distribution, and reproduction in any medium, provided the original work is properly cited.

In order to effectively solve the problems of unreasonable design and low evaluation level in the process of fractal art pattern design and evaluation, a CAD fractal art pattern design and evaluation is proposed based on RBF network algorithm. Through the corresponding sequence sorting of RBF network algorithm, the image surface with the same CAD fractal art pattern design and evaluation attributes is divided into two parts. The main shape in the algorithm is mainly the node of feature tree. According to the later stage of image features, using high-level design basic knowledge and evaluation methods, RBF network algorithm is introduced into the process of original image design modification. Finally, the experimental results show that the algorithm used in this paper has certain advantages in CAD fractal art pattern design and evaluation.

\section{Introduction}

The research and exploration of fractal theory in art design is not enough. It is worth noting that although fractal art has become a more and more important and extensive topic of discussion in the scientific community, this discussion is limited to the technical nature and has not attracted enough attention in the art community. At a deeper level, it only stays in the stage of appreciation and evaluation and lacks indepth analysis, induction, sorting, and summary of the creative principles and ideas of fractal art, not to mention fractal art design theory and its application in design. The appearance of this phenomenon is understandable. Fractal art is first based on the development of computer technology and computer application, and the development speed of computer technology and application far exceeds people's reaction speed. Like any form of artistic design expression in history, the process of artistic design always develops from realizing function to pursuing beauty and value.

With the advancement of globalization, many CAD fractal art patterns do not have their own personality and connotation, so CAD fractal art patterns are activated through cultural factors to make them have their own personality and local characteristics $[1,2]$, which is the main factor in the innovative design of CAD fractal art patterns. The individual characteristics of CAD fractal art pattern have played an important role in CAD fractal art pattern, which greatly improves the competitiveness of CAD fractal art pattern. From the local design of CAD fractal art pattern, on the basis of the characteristics of the original CAD fractal art pattern, in the localization factors of their own characteristics, this will be an important problem to be solved by designers and design institutions. It can make the perfect combination of CAD fractal art pattern and culture [3-5], highlighting the cultural role by using the connotation and quality in creating CAD fractal art pattern, a RBF network algorithm for CAD fractal art pattern design data analysis, which mainly expresses the cultural factors and spreads the form of CAD fractal art pattern to MIG users and studies the measures and methods in cultural creation. This method provides a powerful reference for designers.

According to CAD fractal art pattern to spread the essence of culture and users' cognition of culture, this paper describes the design of CAD fractal art pattern from the perspective of extracting cultural resource graphics and then designs and evaluates CAD fractal art pattern. It improves 
the homogenization of traditional CAD fractal art patterns. Data analysis and development through RBF network algorithm can better establish influence on CAD fractal art pattern.

1.1. RBF Network Algorithm. In order to realize the existing design knowledge of CAD fractal art pattern in high-level design and evaluation, so as to ensure that the algorithm always maintains the original design intention in the process of modification design and evaluation, we have the following.

Assuming that the RBF network algorithm database is separable, the general form of sample $\left(x_{i}, y_{i}\right), i=1,2, \ldots, n$, $x \in R^{d}$ and $y \in\{-1,+1\}$ functions is a specific classification surface equation. $d g(x)=w \cdot x+b$.

$$
w \cdot x+b=0 .
$$

To ensure that all samples can be classified correctly, we must meet

$$
y_{i}[(w \cdot x)+b-1] \geq 0, \quad i=1,2, \ldots n .
$$

To sum up, if the above conditions are met, the minimum classification surface is the best classification surface. The matching of string similarity can transform the solution problem of optimal classification into the following constrained optimization problem, namely, satisfaction formula. Under the constraint of $\|w\|^{2}(2)$, find the function

$$
\varphi(w)=\frac{1}{2}\|w\|^{2}=\frac{1}{2}(w \cdot w) .
$$

Find the minimum value of the function. The problem is further transformed into Lagrange function

$$
\left.L(w, b, a)=\frac{1}{2}(w \cdot w)-\sum_{i=1}^{n} a_{i}\left\{y_{i}\left[w \cdot x_{i}\right] 1+b\right)-1\right\} .
$$

The optimal classification function obtained by solving is $f(x)=\operatorname{sgn}\left\{(w * \cdot x+b *\}=\operatorname{sgn}\left\{\sum_{i=1}^{n} a_{i}^{*} y_{i} \cdot\left(x_{i} \cdot x\right)+b *\right\}\right.$.

$\operatorname{Sgn}()$ is a symbolic function. The exact linear separability of the sample can be processed according to the process of equations (1) to (5). Through the three steps of RBF network algorithm symmetry feature, interactive definition algorithm feature, and automatic creation algorithm feature tree as shown in Figure 1, this paper obtains the advanced design knowledge of CAD fractal art mode and can design and evaluate the searched CAD fractal art mode.

\subsubsection{Symmetrical Features of CAD Fractal Art Patterns.}

The symmetrical feature of CAD fractal art mode is that the point group of one feature in CAD fractal art mode overlaps with the point group of other features through rotation and translation transformation. These two features are considered to be symmetrical features.

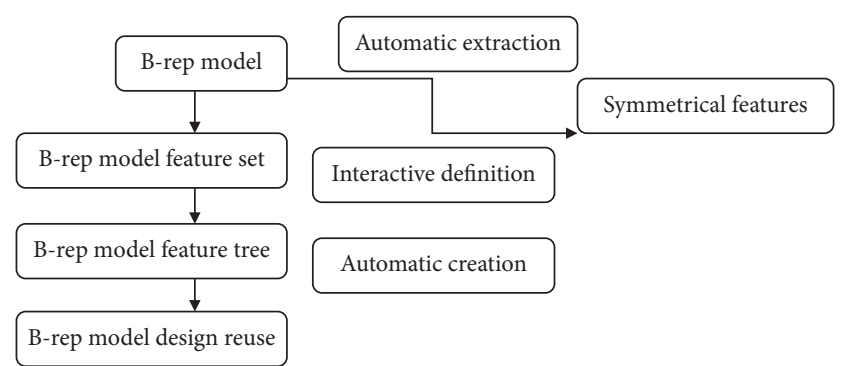

Figure 1: Flowchart of CAD fractal art pattern design and evaluation method.

1.1.2. Interactively Define the Characteristics of CAD Fractal Art Patterns. Some RBF network algorithms can automatically identify the features with symmetric constraints, but for the features of other algorithms, it is difficult to automatically obtain satisfactory features from RBF network algorithms in the current feature recognition methods, so this paper uses the interactive method in the traditional network computing methods to define other features in RBF network algorithms. Interactively select all face sets belonging to a feature and specify the type of the feature.

\subsubsection{Automatically Create Feature Tree of CAD Fractal Art} Pattern. Before automatically creating CAD fractal art pattern feature tree, it is necessary to preprocess the algorithm symmetry characteristics of automatic recognition. The purpose of preprocessing is to reduce the number of feature sets when creating the feature tree by turning the feature sets with the same symmetric constraint into new features and to facilitate the determination of the dependency between feature sets. After obtaining the feature set of RBF network algorithm, because all the features of the algorithm directly or indirectly depend on the main shape features of the algorithm, based on the adjacent relationship between different feature groups, the dependency between features can be determined sequentially and recursively in the root node of the feature tree with the main features of the algorithm, and the feature tree of CAD fractal art pattern can be created.

\section{Basic Concepts and Characteristics of Fractal Art Graphics}

2.1. Basic Concepts of Fractal Art Graphics. Fractal is a kind of art graphics with different rules, fragmented and irregular [6-8]. It is mainly a way with irregular geometric form and complex graphic structure. The fractal art graphics composed of different rules constitute the objective world. This paper presents a CAD fractal art pattern design method based on RBF network algorithm. This method mainly uses the color analysis technology to fully analyze the relevant data, remove the data differences caused by individual data differences, and rely on the contingency caused by data sampling analysis. This method can effectively eliminate the errors caused by human subjectivity. Comprehensively screen the test images to reduce the difference between the 
original image and the compressed image, so as to improve the tester's overall subjective feeling ability. The RBF network algorithm is used for preprocessing, and the superpixel block is treated as a node to improve the color space, reduce the background area during repetition, ensure the accuracy, and greatly reduce the time consumption during the repetition of the algorithm.

\subsection{Characteristics of Fractal Art Graphics}

2.2.1. Self-Similarity. Fractal art graphics look very complex, but changes are tens of millions, but they do not lose integrity. The reason is that they have internal order and seek unity in change. Fractal art graphics have certain similarities between parts and the whole and between parts; that is, they locally reflect the overall structural form and graphic style (as shown in Figure 2). This characteristic seeks change in unity and maintains the unified formal beauty in change, which also reflects the harmonious beauty of the whole and part. This unique self-similarity feature brings great charm to the score graphics, which can produce aesthetic pleasure when people watch.

2.2.2. Infinite Ductility. The self-similarity of fractal art graphics determines its infinite ductility. That is, the fractal structure is infinitely embedded according to its own similarity (as shown in Figure 3). The reason why fractal pattern has a strong visual impact is that each part of the graph is infinitely subdivided according to self-similarity. When the viewer is far sighted, he sees an overall flowing beauty [9-11]. If viewers look closely, they can see the visual beauty with rich details. This infinite nested structure breaks the limitation of the traditional graphic aesthetic form, can be extended infinitely, greatly enriches the structural form of graphics, makes it always full of fantasy artistic charm, and provides a new perspective for the innovation of modern artistic graphics.

\section{Application of Fractal Art Graphics in Graphic Creativity}

3.1. Color Determination of Fractal Art Graphics. The color determination is mainly for the pictures with shadows. Because the shadow parts in the picture are mainly concentrated around the target object, the threshold value is set for a certain kind of picture slice [12-14]. Then, the area exceeding the pixel threshold value is changed to the same value as the background color. Since all the backgrounds used in this design picture are black, the allocation value of the pixels exceeding the threshold value is set to 0. Experimentally determine the pixel value range of the shadow part, set the threshold parameter, and split the pixel value beyond the parameter into the background. Take the orange photo with shadow as the experimental object, read and design a photo, determine the pixel value of the shadow part with the color of the shadow, and eliminate the pixels of the shadow part. The specific discriminant is as follows:

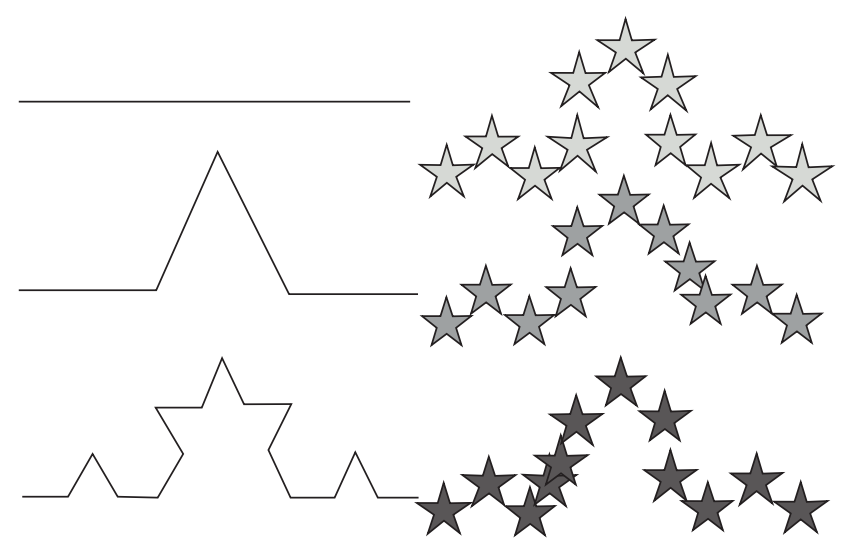

Figure 2: Similarity of fractal art graphics.

$$
d(i, j, u)= \begin{cases}{[0,0,0],} & \text { if } i \geq a \text { and } j \geq a \text { and } u \geq a, \\ {[i, j, u],} & \text { if } i<a \text { and } j<a \text { and } u<a, \\ {[i, j, u],} & \text { if } i>b \text { and } j>b \text { and } u>b\end{cases}
$$

A represents the threshold obtained by experiment, and about 240 values are set. Through B, the edge of the image can be well maintained and the over design of the edge can be avoided. The background of this design is black and the pixel value is black $(0,0,0)$; the color of the shaded part is usually close to white, but the pixel value is greater than any single color other than white. Therefore, the state of the pixel value less than the color value is not changed, the pixel greater than the pixel value is converted into the background, and the target close to the white edge is maintained. Through color determination, the undesigned image can be improved. As shown in Figure 4, in addition to the target edge, the middle shadow can be significantly improved by color determination, but the edge part can be more completely maintained by the limitation of threshold $B$.

CAD fractal art pattern design analysis is defined by diversity and accuracy and comprehensively evaluated to complete the selectivity of CAD fractal art pattern design. Through the analysis of CAD fractal art pattern design and the composition of CAD fractal art pattern design by particle swarm in the search space, the expression of the corresponding CAD fractal art pattern design data information feature vector $\chi_{i}$ is as follows:

$$
l_{\varepsilon}(g)=(1-\rho) l_{\varepsilon}(g-1)+\gamma f\left(\chi_{i}(g)\right) .
$$

Here, $f$ represents the feature data of CAD fractal art pattern design and the corresponding adaptive function of feature vector $\chi_{i} \cdot \gamma \chi_{i}(g)$ represents the fractal art pattern design using CAD software for the $\varepsilon$ time in the process of practical application.

The expression of design $\pi_{p}$ in CAD fractal art pattern design II is

$$
\operatorname{Acu}\left(\pi_{p}\right)=\operatorname{NMI}\left(\pi_{p}, \pi^{*}\right) .
$$

Here, $\pi_{p}$ and $\pi_{q}$ represent the design of CAD fractal art pattern design. If there is less information shared with the 


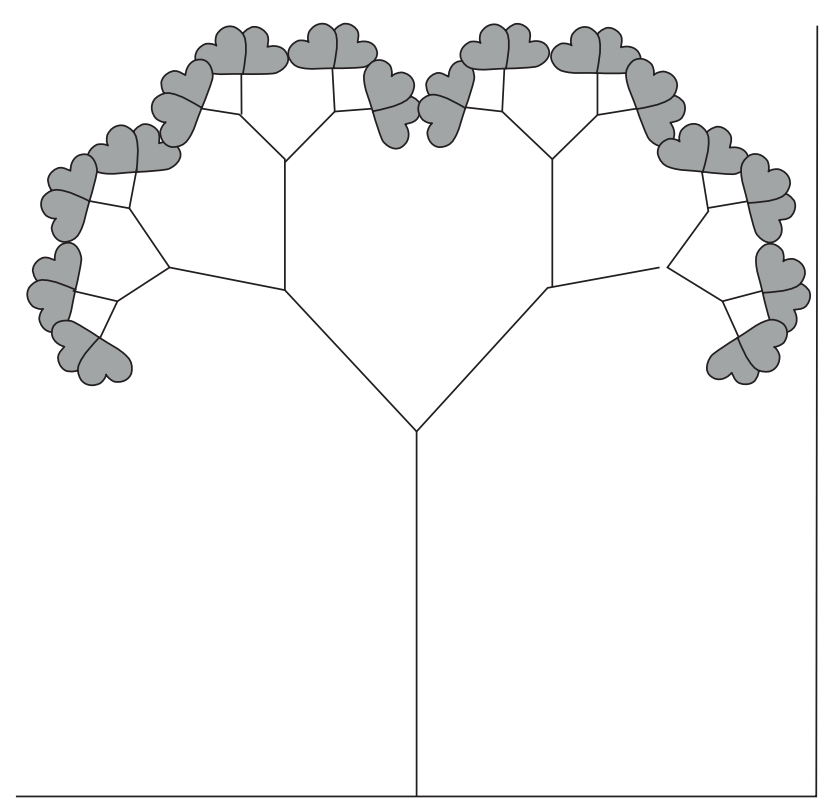

Figure 3: Infinite ductility of fractal art graphics.

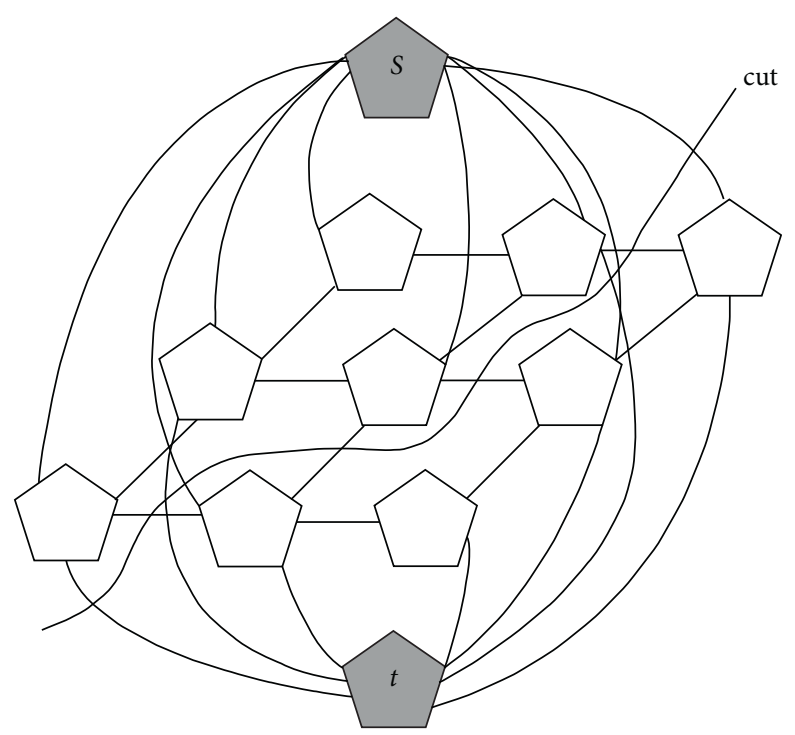

FIgURE 4: RBF network structure. Application of RBF network algorithm in fractal art graphics.

basic cluster of CAD fractal art pattern design, the accuracy of the basic cluster is low, otherwise, vice versa.

Based on the accuracy and diversity characteristics of clusters based on CAD fractal art pattern design, the comprehensive evaluation standard representation of clusters based on CAD fractal art pattern design is defined, including

$$
\operatorname{Eval}\left(\pi_{p}\right)=\lambda \operatorname{Acu}\left(\pi_{p}\right)+(1-\lambda) \operatorname{Div}\left(\pi_{p}\right) .
$$

Here, the correctness and diversity of $\lambda \in[0,1]$ and CAD fractal art pattern design are important in the comprehensive evaluation standard.

Formula (6) calculates the probability $\operatorname{pro}\left(\pi_{p}\right)$ of selecting the basic design algorithm of each CAD fractal art pattern design as the evaluation basic design based on the diversity $\operatorname{Div}\left(\pi_{p}\right)$ of the basic design of CAD fractal art pattern design. The calculation formula is as follows:

$$
\operatorname{pro}\left(\pi_{p}\right)=\frac{\operatorname{Div}\left(\pi_{p}\right)}{\sum_{p=1}^{B} \operatorname{Div}\left(\pi_{p}\right)} .
$$

It is used to randomly select CAD based fractal art pattern design using roulette and obtain CAD fractal art pattern design analysis. Scrambling rearranges the pixel position of the image and only changes the correlation between adjacent pixels of each basic color image but cannot change the histogram of each basic color image [15]. Alternatively, the overall histogram distribution characteristics of the basic color image can be changed by converting the 
pixel values of each basic color image. Therefore, if the pixel value is transformed after scrambling, better design security is obtained.

In this paper, we propose a new three-dimensional chaotic system, which connects Lorenz system and Chen system, but Liu system is only a special example, which is called unified chaotic system.

$$
\left\{\begin{array}{l}
\frac{\mathrm{d} x}{\mathrm{~d} t}=(25 \alpha+10)(y-x), \\
\frac{\mathrm{d} y}{\mathrm{~d} t}=(28-35 \alpha) x-x z+(29 \alpha-1) y, \\
\frac{\mathrm{d} z}{\mathrm{~d} t}=x y-(8-\alpha) \frac{z}{3} .
\end{array}\right.
$$

Here, a system with uniform system parameters within the range of $\alpha \in[0,1]$ has overall chaotic characteristics.

The purpose of the image restoration network module is to ensure that the color and spatial position of each reconstructed pixel can restore the original color and texture of the image to the greatest extent. The total loss function linp of the image restoration network module is defined as shown in (8), which is composed of repair loss of nonmasking area, repair loss of masking area, perception loss, style loss, antiloss, and total variation loss.

$$
L_{\text {total }}^{\text {inp }}=2 L_{\text {valid }}+12 L_{\text {hole }}+0.04 L_{\text {per }}+100\left(L_{\text {style }}^{1}+L_{\text {style }}^{2}\right)+100 L_{\text {adv }}+0.3 L_{\text {var }} .
$$

The weight of each loss term is determined after analyzing the results of 50 independent experiments.

The Manhattan distance between the repaired image and the nonmasked area of the real image is used as the repair loss, where IDAM represents the damaged image, $M$ represents the irregular binary mask (the corresponding area to be repaired in the mask is 0 , and the others are 1), iinp represents the repair result image, and ireal represents the real nondamaged image. The repair loss function of the masked area is shown in (12):

$$
\begin{aligned}
& L_{\text {valid }}=\left\|M \times\left(I_{\text {in } p}-I_{\text {dam }}\right)\right\|_{1}, \\
& L_{\text {hole }}=\left\|(1-M) \times\left(I_{\text {in } p}-I_{\text {dam }}\right)\right\|_{1} .
\end{aligned}
$$

The RBF network algorithm no longer needs to specify the parameter. The algorithm dynamically adjusts the appropriate parameter values based on the texture complexity of different regions of the image, and if the RBF network algorithm maintains a constant speed, the superpixels generated are more neatly unified, which helps to reduce the impact of the shape difference of superpixels on the algorithm in the subsequent processing.

\section{Realization of CAD Fractal Art Pattern Design and Evaluation}

\subsection{Symmetrical Features of CAD Fractal Art Patterns.} Because the faces corresponding to the features of CAD fractal art patterns with symmetric constraints should be the same, that is, they have the same attributes (area and type, etc.), in identifying the features with symmetric relationships in CAD fractal art patterns, in this paper, only the faces with the same attributes are considered, and the time complexity of the algorithm can be greatly reduced. The input of the algorithm in this section is RBF network algorithm, and the output is the feature of symmetric relationship. The symmetric constraints between these features are mainly realized through the following steps.

Step 1. Sort all faces of RBF network algorithm according to their attributes.

Step 2. Classify all faces with the same attributes.

Faces with different attributes may have the same symmetric constraint relationship, so the classes generated in Step 2 need to be merged according to the constraint relationship. Each class after merging includes step 4 of the feature set with the same symmetric constraint relationship, and the symmetric constraint relationship corresponding to each class of feature set is extracted.

The overall complexity of the whole image is calculated by using the image complexity calculation formula. According to the overall complexity of the image, the neighborhood size window with appropriate size is selected as the moving window. Starting from the upper left corner of the image, the image complexity in the neighborhood size box of each pixel is calculated, respectively, and the calculated local complexity is assigned to the corresponding pixel. Thus, the complexity image transformed by complexity calculation can be obtained. In the transformed complexity image, the edge points in the original image will have a large complexity value because of their poor homogeneity and rich gray levels. The complexity of the background region in the original image is further reduced due to its good homogeneity and relatively single gray spatial distribution. In this way, the difference between the region to be segmented and the background region of the original image is strengthened, and it has a good filtering and noise reduction effect due to the local complexity. Therefore, as long as the appropriate complexity value is selected, the target and 
background can be well separated to achieve a good segmentation effect.

These four steps are described in detail as follows.

4.1.1. RBF Network Algorithm Face Sorting. In order to sort the faces in RBF network algorithm, the "size" of the face needs to be defined first. Through NX тм The underlying API function obtains almost all the attributes of the RBF network algorithm face, such as face type, area, ring (ring attribute), edge (edge attribute), vertex, and so on. As an important attribute of RBF network algorithm surface, area can be used to compare the "size" of algorithm surface, but it can not be uniquely determined. Because different types of surfaces may have equal areas, the influence of surface type needs to be considered. At the same time, the number of edges of algorithm surface can also be used to further compare the surfaces of RBF network algorithm. Therefore, this algorithm defines the "size" of RBF network algorithm faces by face type, area, and face edge number and sorts the RBF network algorithm faces: firstly, the RBF network algorithm faces are sorted according to the face type, and then the faces with the same type are further sorted by area and face edge number.

4.1.2. Classify the Faces of the Algorithm. In this paper, the sorted RBF network algorithm is analyzed according to the plane. It can be divided into different sizes and types. These are the same. There may be symmetrical constraints on size and face. In the CAD fractal art mode, because the corresponding surfaces with symmetrical constraints are generally not adjacent, the centered surfaces are further divided through this point, and the adjacency matrix composed of all surfaces (synthetic surfaces) is taken as the zero matrix.

In the CAD fractal art mode shown in Figure 5(a), as shown in Figure 5(b), the class of adjacency matrix with face structure is marked. According to the relationship of adjacency matrix, $c_{i}=\left\{f_{1}, f_{2}, \cdots, f_{8}\right\}$ further classifies the faces to form the composite face $c_{i}$, i.e., $c_{i}=\left\{\left(f_{1}, f_{2}\right),\left(f_{3}, f_{4}\right), \cdots,\left(f_{7}, f_{8}\right)\right\}$, and the critical matrix composed of the combined faces is the zero matrix. The process of further classifying a pair of planes to form a composite surface is actually a subgraph matching process in a graph.

4.1.3. Merge the Faces of RBF Network Algorithm. Class $C=$ $\left\{c_{1}, c_{2}, \cdots, c_{n}\right\}$ processed in Step 2 needs to be further merged, because class $c$ or $c$ may only be a part of a symmetric feature, and $c_{i}$ and $c_{j}$ need to be merged to form the whole symmetric feature. If we do not combine $c_{i}$ and $c_{j}$ but extract symmetry constraints for $c_{i}$ and $c_{j}$, respectively, the extracted symmetry features will be incomplete. Suppose there are two different classes $c_{1}=\left\{f_{1,1}, f_{1,2}, \cdots, f_{1, n}\right\}$ and $c_{2}=\left\{f_{2,1}, f_{2,2}, \cdots, f_{2, m}\right\}$, where $f$ is a face or a composite face. This paper judges whether the two classes can be merged according to the adjacency relationship between the corresponding surfaces of two different $c_{i}$ and $c_{j}$. The specific implementation algorithm is given in RBF network algorithm shown in Algorithm 1.
Although the problem of extracting incomplete symmetrical features can be effectively avoided after the combination of RBF network algorithms of the above algorithms, this paper saves the information before the integration of $\mathrm{RBF}$ network algorithms and determines the problem in the process of user interaction defining other features of the algorithm. If the extracted symmetrical feature is a pseudo feature, the synthetic pseudo feature surface is cancelled.

4.1.4. Extract Symmetric Constraints. Class $C=\left\{c_{1}, c_{2} \cdots, c_{n}\right\}$ of RBF network algorithm surface is merged to form class $C^{\prime}=\left\{c_{1}^{\prime}, c_{2}^{\prime}, \ldots, c_{n}^{\prime}\right\}$; then class $c_{i}^{\prime}$ in $C^{\prime}$ contains the characteristics with the same symmetry constraints. Assuming that there are $n$ identical features in $c_{i}^{\prime}$, calculate the center point set $G=\left\{g_{i}\right\}, 1 \leq i \leq n$ of these $n$ features. The symmetric constraint relationship of these $n$ same features is judged by the distance set $D=\left\{d_{i}\left|d_{i}=\right| g_{i}-g_{c} \mid\right\}$. Among them, $g_{c}=(1 / n) \sum_{i=1}^{n} g_{i}$, as shown in Figure 6(a), the distances of rotationally symmetric features are equal, and the feature distance set of reflection symmetry is shown in Figure 6(b).

For reflection symmetry, the reflection symmetry constraint is obtained by the central point set $G=\left\{g_{i}\right\}$ of $n$ features. Construct covariance matrix.

$$
M_{c}=\left[\begin{array}{c}
g_{1}-g_{c} \\
g_{2}-g_{c} \\
\vdots \\
g_{n}-g_{c}
\end{array}\right]^{T}\left[\begin{array}{c}
g_{1}-g_{c} \\
g_{2}-g_{c} \\
\vdots \\
g_{n}-g_{c}
\end{array}\right] \text {. }
$$

Calculate its eigenvalues and eigenvectors. According to PCA (principal component analysis), the feature vector corresponding to the maximum eigenvalue is the principal axis direction of the center point group $G$. In this specification, if the principal axis direction is defined as the plane normal vector, the symmetry plane of the symmetrical feature can be uniquely determined by the normal vector and the point.

4.2. Automatically Create Feature Tree of CAD Fractal Art Pattern. By automatically extracting symmetric features and defining features interactively with traditional network algorithms, all faces in RBF network algorithm can be formed into different feature set $F$ by automatically extracting symmetric features and interactively defining features with traditional network algorithms. It is assumed that the feature set of RBF network algorithm is $F=\left\{f_{m}, f_{1}^{i}\right\}, 1 \leq i \leq n$, in which $f_{m}$ and $f_{1}^{i}$ are the main shape features and local shape features, respectively. $N$ is the number of local shape features. The feature tree $T$ takes the main feature $f_{m}$ as the initial value and recursively looks for the dependency of the local shape feature $f_{1}^{i}$ step by step until all the features of the feature set $\mathrm{F}$ are effectively added to the feature tree $t$. The pseudocode of this process is as follows:

$$
\begin{aligned}
& T=\left\{f_{m}\right\} . \\
& \text { ParentFeatures }=\left\{f_{m}\right\}
\end{aligned}
$$




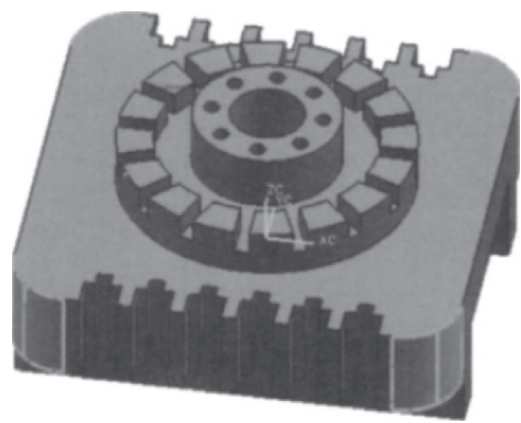

Faces in a class of marked models

(a)

\begin{tabular}{|c|c|c|c|c|c|c|c|c|}
\hline & $f_{1}$ & $f_{2}$ & $f_{3}$ & $f_{4}$ & $f_{5}$ & $f_{3}$ & $f_{7}$ & $f_{8}$ \\
\hline$f_{1}$ & 0 & 1 & 0 & 0 & 0 & 0 & 0 & 0 \\
\hline$f_{2}$ & 1 & 0 & 0 & 0 & 0 & 0 & 0 & 0 \\
\hline$f_{3}$ & 0 & 0 & 0 & 1 & 0 & 0 & 0 & 0 \\
\hline$f_{4}$ & 0 & 0 & 1 & 0 & 0 & 0 & 0 & 0 \\
\hline$f_{5}$ & 0 & 0 & 0 & 0 & 0 & 1 & 0 & 0 \\
\hline$f_{6}$ & 0 & 0 & 0 & 0 & 1 & 0 & 0 & 0 \\
\hline$f_{7}$ & 0 & 0 & 0 & 0 & 0 & 0 & 0 & 1 \\
\hline$f_{8}$ & 0 & 0 & 0 & 0 & 0 & 0 & 1 & 0 \\
\hline
\end{tabular}

Adjacency matrix composed of marked faces

(b)

Figure 5: Classification of algorithm surface. (a) Faces in a class of marked model. (b) Adjacency matrix composed of marked faces.

Construction matrix $M=0$

//The initialization matrix $M$ judges the adjacent relationship of faces in two levels

for $i=1: \mathrm{n}$

for $j=1: \mathrm{m}$

If $f_{1, i}$ and $f_{1, j}$ are adjacent

$$
\mathrm{M}(\mathrm{i}, \mathrm{j})=1 \text {; }
$$

end

end

end

//Judge whether two classes can be merged

if $M \neq 0$

$\mathrm{V} 1=\operatorname{sum}(\mathrm{M}) ; / /$ Sum the columns in $\mathrm{M}$

$\mathrm{V} 2=\operatorname{sum}\left(\mathrm{M}^{\prime}\right) ; / / M^{\prime}$ is the transpose matrix of $\mathrm{M}$. if all elements in $\mathrm{V} 1$ or $\mathrm{V} 2$ are equal

//The adjacency relationship of each face is the same

Merge (c1,c2); //Merge c1, c2 end

end

Algorithm 1: RBF network algorithm.

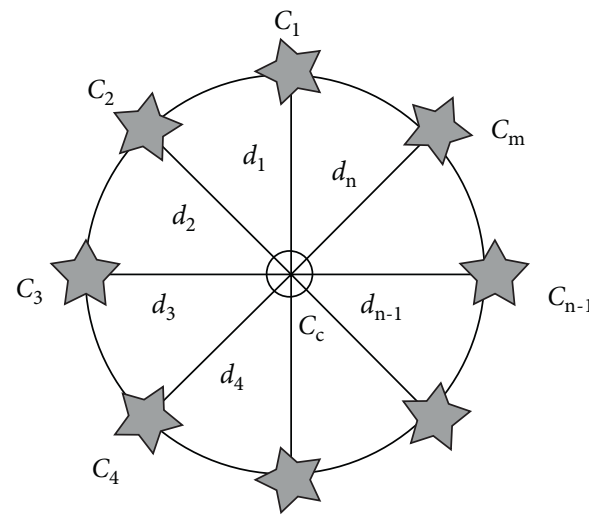

Rotational symmetry feature

(a)

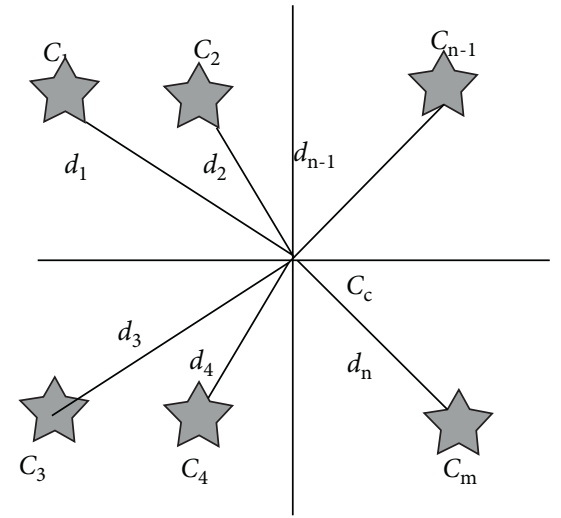

Reflection symmetry feature

(b)

FIGURE 6: The symmetry type of the same feature according to the distance. (a) Rotational symmetry feature. (b) Reflection symmetry feature. 
FreeFeatures $=\left\{f_{1}^{i}\right\}, 1 \leq i \leq n$

While freeFeaturss is not none.

foreach feature $f$ in parentFeaturss.

foreach feature $h$ in freeFeatures

$$
\text { if } \mathrm{h} \longrightarrow \mathrm{f}
$$

T.Add(h)//Add $h$ feature to feature tree

Delete features from freefeatures $\mathrm{H}$

end

end

end

//Updates the current parent feature of the feature tree

Empty parentfeaturss

Add the newly added features in $t$ to parentfeaturss end

4.3. Experiment and Result Analysis. With the development of the information age, the performance of graphics and images has become diversified. People's demand for visual perception has also increased. Fractal pattern stimulates the receiver's visual interest through its unique modeling structure. From the basic form, the fractal pattern simplifies the atmosphere, has strong visual attraction and impact, and skillfully shows the tightness of scientific visual graphics. From the perspective of basic structure, fractal graphics have precise structure and infinite levels, and the overall shape image is irregular and changeable. This structure of fractal brings a great sense of space to the score art graphics. Although fractal graphics have various changes, they seek unity in the changes and always maintain the balance of the picture. Giving dynamic and harmonious beauty goes beyond the static beauty of traditional graphics.

In order to compare the efficiency of the symmetric feature extraction algorithm proposed in this paper, we compare the algorithm with the algorithm in the traditional network algorithm. Table 1 shows some data in the experiment, in which the time required for traditional algorithms to extract symmetrical features from RBF network algorithm is compared. As can be seen from Table 1, the execution efficiency of the algorithm in this paper is significantly better than that in the traditional network algorithm.

If the feature set of RBF network algorithm is $D=\left\{f_{m}, f_{1}^{i}\right\}, 1 \leq i \leq 8$, the feature tree of RBF network algorithm can be automatically created by using the algorithm of constructing feature tree. In the process of automatically creating the algorithm feature tree, this paper judges the dependency between features according to whether there are common edges between feature surface groups. In the first iteration of the algorithm, $f_{m}$ is the parent feature, and it is judged whether the free feature set $f_{1}^{i}(1 \leq i \leq 8)$ depends on $f_{m}$ in turn. After judging that the features $f_{1}^{1}, f_{1}^{3}$, and $f_{1}^{5}$ depend on each other, $f_{1}^{1}, f_{1}^{3}$, and $f_{1}^{5}$ are added to the subfeatures, respectively. The $f_{1}^{1}, f_{1}^{3}$, and $f_{1}^{5}$ are deleted from the free feature set and $f_{1}^{1}, f_{1}^{3}$, and $f_{1}^{5}$ are used as a new parent feature to start a new round of iteration until the free feature set is empty. The algorithm ends, and the feature tree is automatically constructed. The results are shown in Figure 7.

The similarity and infinite extension of fractal art graphics provide the direction of thinking innovation for designers, widen the expression space of modern graphic design, and make people have a better understanding of the coordination and unity of part and whole. In addition to the above graphic creativity, there are also fashion design, napkin design, product surface texture design, many handicraft design, etc. Designers can design artistic expression and artistic creativity through the thinking method of fractal art. For example, in the field of fashion design, if the geometric principles of fractal and function recursion are clearly designed, there will be novel and unique visual aesthetic effects. The graphics of fractal art integrate scientific rationality and artistic sensibility. It is a new form of visual art. Its formation is based on scientific analysis and depends on the design of computer program. Repeat the acceleration command, manual, or semiautomatic process, pause, delete, and overlay the set middle track, and walk in the subtraction and other programs; many fractal patterns with rich shapes will be randomly generated. In order to give people a strong artistic impact, the graphics of fractal art richly express the connotation and form of works with creative thinking through the aesthetic processing of artists. Therefore, the graphics of fractal art make people feel the harmonious beauty of science and art and arouse people's exploration of the incredible charm of the scientific world and the art world.

In order to verify the effectiveness of the proposed algorithm and its effect in practical application, it is compared with the classical traditional algorithm and the improved image design method based on information entropy. The traditional algorithm is used to evaluate the threshold of the image; two design results (a) and (b) in Figure 8 are obtained. Through observation, it is found that the traditional algorithm method does not get good design results, and the area to be designed is not fully evaluated from the image, resulting in obvious underevaluation. The effectiveness and integrity of the image design results are not guaranteed. Through careful observation of (a) and (b) in Figure 8, in the two images, it is found that the part not designed has obvious characteristics. They both have gray values similar to the background of the image and are in a similar position in the gray histogram (b). It is found that the design results have not been significantly improved. Although the information entropy can reflect the content contained in the image and is improved compared with the gray histogram, it still can not describe the spatial distribution information of each gray level in the image, which is an image description method to be improved. 
TABLE 1: Efficiency comparison of two RBF network algorithms and symmetric feature extraction algorithms.

\begin{tabular}{lccc}
\hline RBF network algorithm & Number of faces & Traditional network algorithm (s) & Paper algorithm (s) \\
\hline 1 & 20 & 0.412 & 0.003 \\
2 & 63 & 3.634 & 0.135 \\
3 & 102 & 8.222 & 0.194 \\
4 & 399 & 58.034 & 3.776 \\
\hline
\end{tabular}

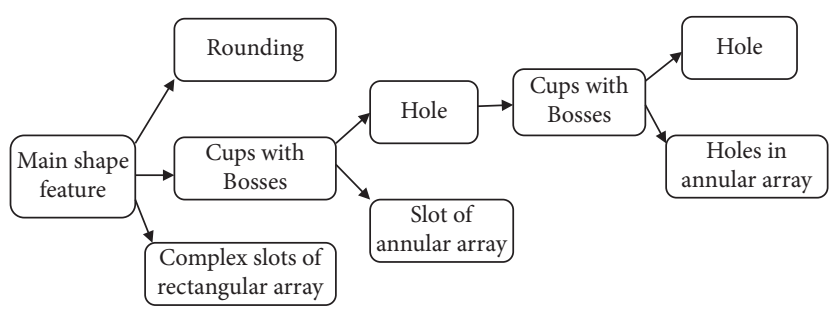

FIgURE 7: Feature tree of automatic creation of CAD fractal art pattern.

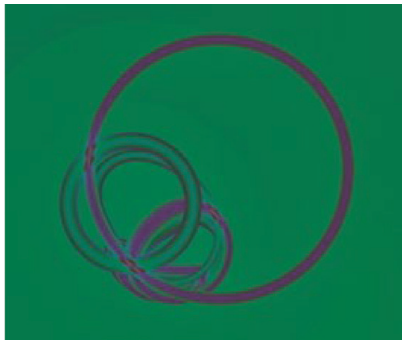

Traditional algorithm

(a)

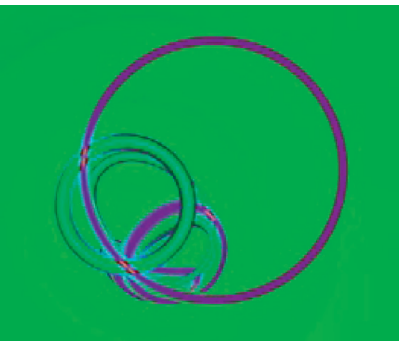

RBF network algorithm

(b)

Figure 8: Comparison of fractal art pattern design and evaluation effects under different algorithms. (a) Traditional algorithm. (b) RBF network algorithm.

\section{Conclusions}

The CAD fractal art pattern design and evaluation method proposed in this paper mainly uses the symmetrical features of CAD images to improve the data. By constructing the CAD fractal art pattern feature tree, we can use the high-level feature schematic diagram, use the computer intelligent and powerful graphics, and use the human-computer interaction function. Finally, by comparing different algorithms, the design of pattern shape and combination is realized, and finally a multishape art pattern is obtained.

\section{Data Availability}

The labeled datasets used to support the findings of this study are available from the corresponding author upon request.

\section{Conflicts of Interest}

The authors declare no conflicts of interest.

\section{Acknowledgments}

This study was sponsored by Henan University of Science and Technology.

\section{References}

[1] W. Wang, G. Zhang, L. Yang, and W. Wang, "Research on garment pattern design based on fractal graphics," EURASIP Journal on Image and Video Processing, vol. 170, no. 1, pp. 545-556, 2019.

[2] Y. Luo, Z. Luo, P. Xu, P. Liu, Y. Du, and J. M. Guo, "Multiperson pose estimation via multi-layer fractal network and joints kinship pattern," IEEE Transactions on Image Processing A Publication of the IEEE Signal Processing Society, vol. 10, no. 2, pp. 174-183, 2018.

[3] R. Fouque, R. Bouclier, J. C. Passieux, and J. N. Pri, "Fractal pattern for multiscale digital image correlation," Experimental Mechanics, vol. 9, no. 2, pp. 95-106, 2020.

[4] B. Biggio, G. Fumera, and F. Roli, "Security evaluation of pattern classifiers under attack," vol. 10, no. 6, pp. 84-88, 2017, https://arxiv.org/abs/1709.00609.

[5] H. Jin and A. Untaroiu, "Elliptical shape hole-pattern seals performance evaluation using design of experiments 
technique," Journal of Fluids Engineering: Transactions of the ASME, vol. 54, no. 1, pp. 376-384, 2018.

[6] Q. Jia, W. D. Xu, J. H. Hu, J. Liu, and L. Y. Zhu, "Design and evaluation of digital camouflage pattern by spot combination," Multimedia Tools and Applications, vol. 79, no. 2, pp. 1-18, 2020.

[7] R. P. Taylor, "Nature's fractal similarities: integrating art and science," Nonlinear Dynamics, Psychology, and Life Sciences, vol. 23, no. 1, pp. 173-176, 2019.

[8] J.-Y. Lee, Y.-S. Chung, D.-S. Kim, G.-H. Bae, J.-S. Bae, and D.-H. Lee, "Development of weft straightener using fabric pattern detection algorithm and performance evaluation," Korean Journal of Computational Design and Engineering, vol. 22, no. 1, pp. 70-79, 2017.

[9] Y. M. Ospina-Dávila and M. Orozco-Alzate, "Parsimonious design of pattern recognition systems for slope stability analysis," Earth Science India, vol. 13, no. 9, pp. 127-133, 2020.

[10] F. Baytar, "Apparel cad patternmaking with 3D simulations: impact of recurrent use of virtual prototypes on students' skill development," International Journal of Fashion Design, Technology and Education, vol. 5, no. 2, p. 14, 2018.

[11] S. Lu, P. Y. Mok, and X. Jin, "A new design concept: $3 \mathrm{~d}$ to $2 \mathrm{~d}$ textile pattern design for garments," Computer-Aided Design, vol. 147, pp. 71-77, 2017.

[12] Z. Lei, "Radiation pattern analysis of reflector antennas using cad model based physical optics method," IEEE Access, vol. 6, no. 5, pp. 744-746, 2019.

[13] M. S. Harsha, M. Praffulla, M. R. Babu, G. Leneena, T. S. Krishna, and G. Divya, "The effect of cavity design on fracture resistance and failure pattern in monolithic zirconia partial coverage restorations - an in vitro study," Journal of Clinical and Diagnostic Research:Journal of Clinical and Diagnostic Research, vol. 11, no. 5, pp. ZC45-48, 2017.

[14] K. Reuter and J. Köfinger, "Cadishi: fast parallel calculation of particle-pair distance histograms on cpus and gpus," Computer Physics Communications, vol. 17, no. 5, pp. 198-207, 2018.

[15] A Goldbach, A. M. Bauer, W. Roland, and K. w. Bletzinger, "Der $\mathrm{cad}^{-\cdot-}$ ntegrierte parametrische entwurfsprozess von membrantragwerken," Stahlbau, vol. 89, no. 12, pp. 2449-2469, 2020. 